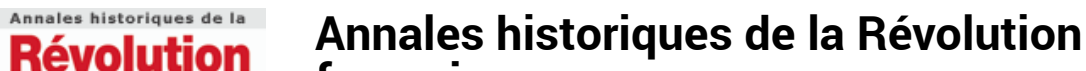

française française

344 | avril-juin 2006

La prise de parole publique des femmes

\section{Les officiers de marine du premier Empire}

Josiane Bourguet-Rouveyre

\section{(2) OpenEdition \\ Journals}

Édition électronique

URL : https://journals.openedition.org/ahrf/6782

DOI : 10.4000/ahrf.6782

ISSN : 1952-403X

Éditeur :

Armand Colin, Société des études robespierristes

Édition imprimée

Date de publication : 1 juin 2006

Pagination : 274-277

ISSN : 0003-4436

Référence électronique

Josiane Bourguet-Rouveyre, "Les officiers de marine du premier Empire », Annales historiques de la Révolution française [En ligne], 344 | avril-juin 2006, mis en ligne le 02 juillet 2008, consulté le 23 avril 2022. URL : http://journals.openedition.org/ahrf/6782 ; DOI : https://doi.org/10.4000/ahrf.6782

Ce document a été généré automatiquement le 23 avril 2022.

Tous droits réservés 


\title{
Les officiers de marine du premier Empire
}

\author{
Josiane Bourguet-Rouveyre
}

\section{RÉFÉRENCE}

Pierre LÉVÊQUE, Les officiers de marine du premier Empire, Paris, Service historique de la Marine nationale, 2003, 2 vol., 654 p., ISBN 2-11-091844-6, $30.49 €$.

1 L'ouvrage publié par le Service historique de la Marine résulte d'une thèse de l'Université Paris 1, soutenue en 1998. La marine, sous le Consulat et l'Empire, est apparue à bien des égards comme la mal aimée, à défaut d'être le parent pauvre des forces militaires de l'époque. Il est vrai que les désastres navals, face à une Angleterre en tous points supérieure, ont sinon occulté, du moins minimisé les succès plus modestes, remportés le plus souvent dans des conditions difficiles, et relégué au second plan le courage et le dévouement d'officiers et de marins engagés dans des opérations parfois désespérées. On a aujourd'hui largement démontré que Napoléon n'a pas négligé la marine et qu'après Trafalgar, des efforts tout particuliers ont visé à reconstruire une flotte susceptible de contester la suprématie maritime anglaise, sans y parvenir faute de moyens, de temps et peut-être aussi d'expérience, à cause d'une activité très réduite. Aussi n'est-ce pas sur ces aspects que Pierre Lévêque revient. Son intention est de faire une étude sociale du milieu des officiers de marine, inscrivant ses recherches dans un cadre initié par Gilbert Bodinier pour l'armée de terre à l'époque napoléonienne, ou encore Michel Vergé-Franceschi, Martine Acerra et Alain Cabantous pour la marine d'Ancien Régime. Étudier sur un plan socio-culturel l'ensemble des officiers de marine supposait la connaissance d'un corpus immense dépassant largement le cadre d'une thèse d'université. Aussi est-ce raisonnablement que l'auteur a délimité son propos à un groupe particulier : les quelque cinq cents lieutenants de vaisseau en service en 1811, embarqués, en mission dans les ports ou encore en captivité. Il a épluché leurs dossiers et la correspondance du Ministère de la Marine, 
tout en enrichissant ses apports archivistiques de la compulsion de plusieurs mémoires du temps, parmi lesquels le précieux témoignage de Jurien de la Gravière.

2 Le corps des officiers de marine a été profondément modifié pendant la Révolution et les conséquences en sont visibles en 1811. Les officiers issus du grand corps ne représentent plus qu'un nombre infime et résiduel de l'ensemble; quelques-uns sont d'anciens émigrés. Les plus nombreux sont venus du commerce, à l'époque où le gouvernement révolutionnaire a fait appel à eux pour répondre à l'effort de guerre : ils forment presque la moitié de l'effectif. Il y a aussi les promus, anciens marins devenus enseignes sous la Révolution, et passés lieutenants sous le Consulat ou l'Empire. Il y a enfin les plus jeunes, ceux qui ont commencé leur carrière sous le Directoire et qui constituent un groupe étoffé de jeunes lieutenants de vaisseau, tout juste trentenaires. Ce corps présente donc une certaine hétérogénéité d'origine, mais montre une remarquable homogénéité par l'esprit, homogénéité consciencieusement entretenue par les ministres qui se succèdent à la Marine : Truguet sous le Directoire, Forfait, puis Decrès, sous le Consulat et l'Empire. Persuadés, comme d'ailleurs Bonaparte, qu'il y a un lien entre origine familiale et sens de l'honneur, ils ont à cœur de recruter des officiers de qualité, pris de préférence dans les familles de notables bourgeois ou appartenant à l'ancienne noblesse. Ils partagent les mêmes préjugés à l'égard des officiers venus de la marine marchande, "la plus mauvaise éducation qu'un marin militaire puisse avoir ", selon Decrès, cité par l'auteur, préjugé pourtant fréquemment démenti par les faits. Il en résulte que d'une façon générale, et si l'on excepte les anciens marins, les officiers de marine sont issus de milieux aisés liés à la mer ou à la chose militaire : ancienne noblesse, militaires, marchands-fabricants, officiers civils. La Révolution a modifié les aires géographiques de recrutement, nous montre Pierre Lévêque, en les diversifiant. Certes, la Bretagne et la Provence fournissent toujours leur lot, mais elles ont vu leur part sensiblement se rétrécir au profit de la CharenteMaritime ou des ports de la Manche et de la Mer du Nord. Quant aux jeunes officiers, ils sont originaires pour les trois-quarts d'entre eux des départements intérieurs, et bon nombre sont des Parisiens, attestant la diversification des vocations et le décalage entre celles-ci et l'origine géographique. Pourtant Pierre Lévêque souligne l'importance de la pratique dans la formation et n'évoque que très rapidement le passage de certains officiers - les plus jeunes - dans une école militaire comme l'éphémère École de Mars et surtout l'École polytechnique, sans mentionner les nombreuses écoles de navigation qui existent, sous l'Empire, dans tous les grands ports de Méditerranée ou de la façade atlantique, qu'il s'agisse de Toulon et Marseille, ou de Bordeaux, Rochefort, Lorient, Nantes, Dunkerque ou Anvers. Est-ce à dire qu'aucun des lieutenants étudiés n'est passé par là? On reste également un peu sur sa faim, lorsque l'auteur aborde la question du bagage culturel des officiers : celui-ci n'est mesuré qu'à la connaissance de la littérature ou du latin, sans prendre en compte la culture scientifique que certains avaient pu acquérir par les aspects théoriques de leur formation. Si l'auteur insiste sur l'existence d'un examen de recrutement et l'organisation de cours pour s'y préparer, on sait peu de chose des programmes ou des aptitudes intellectuelles et techniques exigées, en dehors de la simple maîtrise de l'écrit.

3 Les carrières sont relativement rapides. Il faut entre deux et quatre ans à un aspirant pour devenir officier, mais il y a d'importantes disparités selon que l'on est ancien du commerce (promotions très rapides sous la Révolution), ancien marin ou jeune aspirant. Le rythme de carrière prend parfois en compte l'origine sociale, mais le plus 
souvent les seuls mérites et talents, ou les actes de bravoure. Néanmoins, on ne saurait négliger, souligne l'auteur, le clientélisme qui, dans cette arme comme dans les autres, joue un rôle important. Les protecteurs accélérateurs de carrière sont des officiers supérieurs de diverses armes, des hommes politiques, particulièrement sous le Directoire, des membres de la famille impériale à l'époque napoléonienne, des représentants du Corps législatif ou du Sénat, des ministres ou de hauts fonctionnaires. Il ne s'agit donc pas d'un clientélisme exclusivement militaire, et il renvoie à des pratiques très développées dans d'autres pays européens, par exemple en Angleterre. Ces disparités ne paraissent pas affecter la solidarité qui lie les officiers entre eux, par delà les jalousies ou les ambitions concurrentes. Et même si la fraternité, que préconisaient certains textes révolutionnaires entre officiers et marins, n'a jamais été vraiment à l'ordre du jour, les actes d'indiscipline, les mutineries sont très rares : un seul cas est mentionné par l'auteur, en 1809, mettant en scène un ex-officier de corsaire tentant de s'emparer du navire sur lequel il était embarqué, afin de se livrer de nouveau à la guerre de course. Les autres incidents signalés ne portent que sur les approvisionnements, ou le respect des règles internationales, pas toujours admises par les marins, comme lorsqu'on leur défend de chercher à s'emparer de la cargaison d'un bâtiment neutre. Cela n'empêche cependant pas certains officiers de pratiquer une contrebande très active et presque assurée de l'impunité, même au plus fort du blocus continental.

4 Toute une partie de l'ouvrage est consacrée à la vie en mer et dans les ports, ainsi qu'aux effets de la guerre. La plupart des officiers préfèrent naviguer et affronter les risques du métier, plutôt que de rester au port où les tâches sont fastidieuses. La vie en mer a son lot de dangers: maladies comme le scorbut dont on commence à bien connaître les antidotes, en embarquant citrons, moût de bière fermentée ou cochléaria, les affections pulmonaires, les rhumatismes, les ophtalmies, les maladies tropicales comme la fièvre jaune; blessures consécutives aux engagements souvent très meurtriers ; enfin la captivité sur les fameux pontons anglais ou espagnols. En 1811, sur le corpus étudié par Pierre Lévêque, il y a 79 prisonniers qui ne seront libérés qu'en 1814. En effet, libérations et échanges sont très rares avant cette date, essentiellement parce que les prisonniers faits par les Anglais étant plus nombreux que ceux des Français, l'échange aurait été disproportionné. Quelques officiers seulement, comme Jurien de la Gravière, ont été libérés sur parole. La vie privée des officiers de marine est soumise au contrôle de l'administration. Ils doivent ainsi demander l'autorisation de se marier et, dans ce cas, les autorités maritimes civiles ou militaires se livrent à des vérifications concernant la famille et les vertus de la future épouse. Des observations sont demandées aux préfets maritimes, et le ministre peut refuser telle ou telle prétendante, au principe de la mésalliance que pourrait constituer une union socialement mal assortie. "Une certaine fortune est indispensable à un bon mariage ", affirme Decrès en 1811. Il en résulte une forte endogamie. L'âge moyen au mariage des lieutenants de vaisseau est élevé, 35 ans, ce qui n'a rien d'étonnant. Pierre Lévêque cite encore Jurien de la Gravière expliquant ses longues hésitations avant d'épouser la femme qu'il aimait: "Jusqu'alors je ne m'étais point senti le courage de lui offrir ce triste don, gage de tant d'inquiétude et de souffrance, qu'on appelle l'amour d'un marin ».

5 Enfin, l'auteur s'attaque à un sujet bien délicat: celui des attitudes politiques des lieutenants de vaisseau, attitudes qu'il essaie de cerner au travers de très succincts renseignements. En annexe à l'ouvrage, il dresse un tableau nominatif de ces officiers, 
indiquant le déroulement chronologique de leur carrière, leur situation en 1811 et une caractéristique politique concise : républicain, bonapartiste, royaliste. Il s'en dégage l'impression que la grande majorité de ces officiers ont été de fidèles serviteurs du régime impérial, et que certains ont même été particulièrement attachés à la personne de Napoléon en qui ils voyaient l'héritier de la Révolution, une attitude confirmée par la façon dont ils accueillent l'annonce de l'entrée des alliés à Paris en 1814, en voulant se former en un bataillon de volontaires. Contrairement à ce qui s'est passé dans l'armée de terre, il n'y eut aucune conspiration antinapoléonienne dans la marine. L'ouvrage est agrémenté de quelques illustrations et surtout de précieux diagrammes et figures sur les divers aspects statistiques évoqués dans la thèse. Ils éclairent un propos parfois un peu anecdotique, mais vivant et concret. 